\title{
A NUMERICAL STUDY OF THE LIMITED MEMORY BFGS METHOD AND THE TRUNCATED-NEWTON METHOD FOR LARGE SCALE OPTIMIZATION*
}

\author{
STEPHEN G. NASH ${ }^{\dagger}$ AND JORGE NOCEDAL ${ }^{\ddagger}$
}

\begin{abstract}
This paper examines the numerical performances of two methods for large-scale optimization: a limited memory quasi-Newton method (L-BFGS), and a discrete truncated-Newton method (TN). Various ways of classifying test problems are discussed in order to better understand the types of problems that each algorithm solves well. The L-BFGS and TN methods are also compared with the Polak-Ribière conjugate gradient method.
\end{abstract}

Key words. large scale nonlinear optimization, limited memory method, truncated-Newton method, conjugate-gradient method

AMS(MOS) subject classifications. 65,49

1. Introduction. Suppose we had to solve an unconstrained optimization problem,

$$
\min f(x),
$$

where $f$ is a smooth function, the number of variables $n$ is large, and a subroutine to evaluate $f(x)$ and $\nabla f(x)$ is available. What method should we choose?

The answer will depend on how much knowledge we have about the structure of the objective function, and on the size of the problem. Newton's method using sparse matrix estimation techniques [2], and the partitioned quasi-Newton method of Griewank and Toint [8] can be highly efficient if sufficient information is supplied to the algorithms. However, it is sometimes too difficult for the user to provide this information, and efficient and reliable software that supplies it automatically is not yet available. In addition, the storage and arithmetic costs for these methods can be prohibitive if the Hessian matrix is not very sparse, or if the problem is extremely large. In these cases, it is better to use other, less ambitious, algorithms. The limited memory BFGS method (L-BFGS) and the discrete truncated-Newton method (TN) represent two classes of methods in this category. They use a low and predictable amount of storage, and only require the function and gradient values at each iterateand no other information about the problem. Both methods have been tested on large problems and their performance appears to be satisfactory.

In this paper we study the relative performance of L-BFGS and TN on a set of 45 large test problems with a number of variables ranging from 100 to 10,000 . Our goal is to study the two methods in a controlled environment to highlight the differences between them and to indicate to the reader the types of problems that are well suited to each algorithm. Our study is limited by the collection of test problems we have used; however, we have tried to produce a test set of considerable breadth. Some test problems arise from applications, whereas others are artificial. Some of the

* Received by the editors January 10, 1990; accepted for publication (in revised form) January 8 , 1991.

$\dagger$ Operations Research and Applied Statistics Department, George Mason University, Fairfax, Virginia 22030.

$\ddagger$ Department of Electrical Engineering and Computer Science, Northwestern University, Evanston, Illinois 60208. This author was supported by the Applied Mathematical Sciences subprogram of the Office of Energy Research, U.S. Department of Energy, under contract DE-FG0287ER25047-A001, and by National Science Foundation grant ASC 87-19583. 
problems are quadratic or nearly quadratic, while others are highly nonlinear. Some are nonconvex. There are varying degrees of ill-conditioning. Our conclusions can only be based on this sample of results, but we hope that they may be more generally useful - both to readers who solve practical problems, and to readers who develop and test new algorithms.

Many limited memory methods have been proposed; some resemble the conjugategradient method, and others, the BFGS method. We chose the L-BFGS method because the studies of Gilbert and Lemaréchal [4] and Liu and Nocedal [10] seem to indicate that it is the best limited memory method available to date. Several implementations of the truncated-Newton method have also been proposed, but few codes are as developed as that of Nash [13], which we have chosen for our tests.

Since the authors of the present paper have been closely involved in the development of the methods tested here, a careful effort was made to run them in their best form. This probably also avoided a conscious or unconscious bias towards one of the methods, while setting the test problems or while interpreting the results. The two codes used for this study are not new; they have both been refined during the last years, and neither was especially modified for these runs. We were aware of the dangers of "tuning" methods to a set of test problems. As a result, the nature of the computational tests was specified in detail in advance: A single, fixed version of each algorithm was provided, with all parameters such as tolerances and stopping conditions chosen beforehand. The set of test problems was also chosen in advance, and a single run on each test problem was made. (One exception had to be made. On three runs, the algorithms were unable to satisfy our prespecified convergence tolerance. These three problems were run again with a slightly less stringent convergence tolerance.)

Our results indicate that, for L-BFGS and TN, two properties of the problems can be used to make an informed decision on the choice of method. These are the cost of the function-gradient evaluation and the "degree of nonlinearity" of $f$ (this latter property is defined more precisely in $\S 4$ ).

What follows is a brief outline of the paper. The L-BFGS and TN algorithms are described in $\S 2$; the test problems and numerical results are presented in $\S 3$. The analysis of the test problems, their classification into types, and the correlation of these types with the two methods, appear in $\S \S 4$ and 5 . In the final part of the paper, we compare the two methods to the Polak-Ribière conjugate-gradient method, to obtain another measure of their efficiency.

2. Description of the TN and L-BFGS methods. The truncated-Newton algorithm and the limited memory BFGS method used in this study have already been published, and hence we will only describe them in outline. The L-BFGS has been incorporated into the Harwell Library under the name VA15, and is described by Liu and Nocedal [10]. The TN method is described by Nash [13] (a more precise description of many parts of the method is in Nash [14]). We now give a brief description of their major components.

2.1. The truncated-Newton algorithm. At each "outer" iteration, an approximate solution is found to the Newton equations

$$
G_{k} p_{k}=-g_{k},
$$

where $g_{k}=g\left(x_{k}\right)=\nabla f\left(x_{k}\right)$ is the gradient at the $k$ th iteration, and $G_{k}=\nabla^{2} f\left(x_{k}\right)$ is the Hessian. This is done using an "inner" iteration based on a preconditioned linear 
conjugate-gradient method [3]. If indefiniteness in the Hessian is detected, the inner iteration is terminated. The approximate solution $p_{k}$ (the search direction) is then used in a linesearch to get a new point $x_{k+1}=x_{k}+\alpha_{k} p_{k}$ where $f\left(x_{k+1}\right)<f\left(x_{k}\right)$ and $\alpha_{k}>0$. More precisely:

(1) The outer iteration is terminated when

$$
\left\|g\left(x_{k}\right)\right\|_{\infty}<10^{-6}\left(1+\left|f\left(x_{k}\right)\right|\right) .
$$

(2) The linesearch was performed using the iteration described by Gill and Murray [6]. It is based on cubic interpolation, and is terminated when

$$
\left|g\left(x_{k}+\alpha_{k} p_{k}\right)^{T} p_{k}\right| \leq \eta\left|g\left(x_{k}\right)^{T} p_{k}\right|
$$

and

$$
f\left(x_{k}\right)-f\left(x_{k}+\alpha_{k} p_{k}\right) \geq-\mu \alpha_{k} p_{k}^{T} g\left(x_{k}\right),
$$

with $\eta=0.25$ and $\mu=10^{-4}$. An initial guess of $\alpha_{k}=1$ is used. We refer to (2.3)-(2.4) as the strong Wolfe conditions.

(3) The conjugate-gradient inner iteration is preconditioned by a scaled two-step limited memory BFGS method, with Powell's restarting strategy used to reset the preconditioner periodically. It is based on formula (6.5) in [6]: Let $t$ be the iteration where the last restart occurred. Define $s_{1}=x_{k}-x_{t}, y_{1}=g_{k}-g_{t}$, $s_{2}=x_{k}-x_{k-1}, y_{2}=g_{k}-g_{k-1}$, and let $D_{k}$ be a scaling matrix. Then the approximation $H_{k}$ to the Hessian is obtained via

$$
U=\Gamma_{\mathrm{BFGS}}\left(D_{k}, y_{1}, s_{1}\right), \quad H_{k}=\Gamma_{\mathrm{BFGS}}\left(U, y_{2}, s_{2}\right),
$$

where $\Gamma_{\mathrm{BFGS}}(A, y, s)$ represents the formula for the BFGS update initialized with matrix $A$, and with vectors $y$ and $s$ corresponding to the change in the gradient and parameter vectors, respectively. The scaling matrix $D_{k}$ used here is a diagonal approximation to the Hessian obtained by BFGS updating; see equation (10) in Nash [14]. This reference also describes the preconditioner in more detail. We note, in particular, that safeguarding is needed to ensure that the scaling matrix is sufficiently positive definite.

(4) The matrix-vector products required by the inner conjugate-gradient algorithm are obtained by finite differencing [19]. Given a vector $v$, the product $G_{k} v$ is approximated by

$$
G_{k} v \approx\left[g\left(x_{k}+\delta v\right)-g_{k}\right] / \delta
$$

where $\delta=\left(1+\left\|x_{k}\right\|_{2}\right) \sqrt{\epsilon}$, and $\epsilon$ is the relative machine precision (the "machine epsilon").

(5) The inner algorithm is normally terminated when

$$
i\left(1-\frac{Q_{k}\left(p_{i-1}\right)}{Q_{k}\left(p_{i}\right)}\right) \leq 0.5
$$

where $i$ is the counter for the inner iteration, $p_{i}$ is the $i$ th approximation to the search direction, and $Q_{k}(p)=\frac{1}{2} p^{T} G_{k} p+p^{T} g_{k}$ is the value of the quadratic model. This test is explained further in [16]; it guarantees a linear rate of convergence for the optimization algorithm, and detects convergence of the 
inner iteration. An upper limit of 50 inner iterations is imposed (this limit was encountered on five of the problems). In addition, the inner algorithm is terminated whenever nonpositive-definiteness is detected in the Hessian. In this case, $p_{k}$ is the iterate obtained prior to detecting indefiniteness.

The truncated-Newton method requires storage for 16 vectors of length $n$ (the vectors $x_{k}, g\left(x_{k}\right)$, and 14 work vectors). Each outer iteration consists of three stages: setup, computation of the search direction, and the linesearch. These costs are listed in Table 1. The typical cost was obtained by assuming 1 iteration in the linesearch and 5 inner iterations; these are typical values observed in our test runs.

TABLE 1

Cost of truncated-Newton iteration.

\begin{tabular}{lrr|r}
\hline Operation & Flops & f-g & Storage \\
\hline Setup & $81 n$ & 0 & \\
Search direction iteration & $48 n$ & 1 & \multirow{2}{*}{$16 n$} \\
Line search iteration & $4 n$ & 1 & \\
\hline Typical cost & $325 n$ & 6 & \\
\hline
\end{tabular}

Here "Flops" denotes additions, multiplications, or divisions, and "f-g" refers to the number of function-gradient evaluations. The setup is performed once per outer iteration. The search direction and linesearch iterations are repeated until appropriate convergence tests are satisfied, although the linesearch is frequently terminated after only one iteration. Note that each inner iteration requires one gradient evaluation to estimate the matrix-vector product (2.6). The number of inner iterations can vary greatly from problem to problem; thus large deviations from this "typical cost" occur in practice. On the test problems in this paper, where an upper limit of 50 inner iterations was imposed, the number of inner iterations varied between 1 and 50 with both extremes achieved.

2.2. The limited memory BFGS method. We view the L-BFGS method as an adaptation of the BFGS method to large problems, and the implementation of both methods is very similar. In the BFGS method, the approximation $H_{k}$ to the inverse Hessian matrix of $f$ is updated by

$$
H_{k+1}=V_{k}^{T} H_{k} V_{k}+\rho_{k} s_{k} s_{k}^{T},
$$

where

$$
V_{k}=I-\rho_{k} y_{k} s_{k}^{T},
$$

$s_{k}=x_{k+1}-x_{k}, y_{k}=g_{k+1}-g_{k}$, and $\rho_{k}=1 / y_{k}^{T} s_{k}$. The search direction is given by

$$
p_{k+1}=-H_{k+1} g_{k+1} \text {. }
$$

We say that $H_{k+1}$ is obtained by applying one BFGS correction to $H_{k}$. In the L-BFGS method, instead of forming the matrices $H_{k}$, we save the vectors $s_{k}$ and $y_{k}$ that define them implicitly. We choose a number $m$ of corrections that we wish to store, and at the $k$ th iteration (with $k>m$ ) proceed as follows:

(1) Define the diagonal matrix

$$
H_{k}^{0}=\frac{y_{k}^{T} s_{k}}{\left\|y_{k}\right\|_{2}^{2}} I
$$

where $I$ denotes the identity matrix. This scaling is suggested by Oren and Spedicato [20]. 
(2) Obtain $H_{k}$ by applying $m$ BFGS corrections to $H_{k}^{0}$, using the $m$ previous vectors $s_{i}$ and $y_{i}$. Compute the product $H_{k} g_{k}$ using the recursive formula described in $\S 4$ of [18]. This formula takes advantage of the symmetry of the BFGS updating to reduce the number of arithmetic operations.

(3) Perform a linesearch along the direction $p_{k}$, enforcing the strong Wolfe conditions (2.3)-(2.4), and trying the steplength $\alpha_{k}=1$ first. We use the values $\eta=0.9$ and $\mu=10^{-4}$. The linesearch is performed by means of the routine CVSRCH of Moré and Thuente [11], which uses cubic interpolation.

(4) The iteration is finished when (2.2) is satisfied.

We note that once $m$ is chosen, the only parameters in the L-BFGS method are the constants $\eta$ and $\mu$. The simple scaling of step 1 contributes significantly to the efficiency of the method. Our experience indicates that values of $m$ in the range $3 \leq m \leq 7$ give the best results, and in this paper we use the value $m=5$. The L-BFGS method requires $2 m(n+1)+4 n$ storage locations. Table 2 gives the storage and computational requirements of the L-BFGS method, assuming $m=5$. We have observed that L-BFGS requires an average of 1.2 iterations within the linesearch; using this value we obtained the typical cost of the iteration, given in Table 2 .

TABLE 2

Cost of the limited memory BFGS iteration.

\begin{tabular}{lrr|r}
\hline Operation & Flops & f-g & Storage \\
\hline Setup & $4 n$ & 0 & \\
Search direction computation & $33 n$ & 0 & \multirow{2}{*}{$14 n$} \\
Linesearch iteration & $4 n$ & 1 & \\
\hline Typical cost & $42 n$ & 1.2 & \\
\hline
\end{tabular}

Except for the first $m-1$ iterations, the cost of computing the search direction in the L-BFGS method is uniform and predictable; it is a function of $m$ and $n$.

Comparing Tables 1 and 2, we see that the storage requirements of TN and LBFGS (with $m=5$ ) are very similar, and that the arithmetic costs are drastically different. TN uses an elaborate, variable-cost iteration with partial second-derivative information, whereas L-BFGS uses a fixed, low-cost formula requiring no extra derivative information. In fact, TN contains a limited memory method, since the preconditioner used for the inner iteration is similar to an L-BFGS matrix with $m=2$, and several multiplications with this matrix are performed in one iteration. $\mathrm{TN}$ and L-BFGS therefore use different principles to compute search direction. It should be noted that the routines used for the linesearch are similar: both use cubic interpolation to obtain the strong Wolfe conditions (only the value of the parameter $\eta$ in (2.3) is different).

3. Numerical tests. All the problems used in our tests have been described elsewhere; approximately half of them were used by Liu and Nocedal in their study of L-BFGS [10]. Table 3 lists the problems and the number of variables used for the runs, and gives references to detailed descriptions of the test functions and starting points. For test problems 8,9 , and 10, starting point 3 from the reference was used. The problems are not numbered consecutively because they belong to a larger collection of test problems to which we may want to refer in future studies. The number of variables in the test set ranges from 100 to 10,000 , and, as will be seen in $\S 4$, the problems form a varied grouping. We verified that, in each run, both methods converged to the same solution point.

The results of the tests are given in Tables 4 and 5. There "It" and "f-g" record 
TABLE 3

List of test functions

\begin{tabular}{llll}
\hline Problem & Name & Reference & $n$ \\
\hline 1 & Calculus of variations 1 & Gill and Murray [5] & 100,200 \\
2 & Calculus of variations 2 & Gill and Murray [5] & 100,200 \\
3 & Calculus of variations 3 & Gill and Murray [5] & 100,200 \\
6 & Generalized Rosenbrock & Moré, Garbow, and Hillstrom [12] & 100,500 \\
8 & Penalty 1 & Gill and Murray [6] & 100,1000 \\
9 & Penalty 2 & Gill and Murray [6] & 100 \\
10 & Penalty 3 & Gill and Murray [6] & 100,1000 \\
28 & Extended Powell singular & Moré, Garbow, and Hillstrom [12] & 100,1000 \\
29 & Variably dimensioned & Moré, Garbow, and Hillstrom [12] & 100,500 \\
31 & Brown almost linear & Moré, Garbow, and Hillstrom [12] & 100,200 \\
38 & Tridiagonal 1 & Buckley and LeNir [1] & 100,1000 \\
39 & Linear minimal surface & Toint [23] & 121,961 \\
40 & Boundary-value problem & Toint [23] & 100 \\
41 & Broyden tridiagonal nonlinear & Toint [23] & 100 \\
42 & Extended ENGVL1 & Toint [23] & $1000,10,000$ \\
43 & Ext. Freudenstein and Roth & Toint [23] & 100,1000 \\
45 & Wrong extended Wood & Toint [23] & 100 \\
$46(1)$ & Matrix square root $(n s=1)$ & Liu and Nocedal [9] & 100 \\
$46(2)$ & Matrix square root $(n s=2)$ & Liu and Nocedal [9] & 100 \\
47 & Sparse matrix square root & Liu and Nocedal [9] & 100,1000 \\
48 & Extended Rosenbrock & Moré, Garbow, and Hillstrom [12] & $1000,10,000$ \\
49 & Extended Powell & Moré, Garbow, and Hillstrom [12] & 100,1000 \\
50 & Tridiagonal 2 & Toint [23] & 100,1000 \\
51 & Trigonometric & Moré, Garbow, and Hillstrom [12] & 100,1000 \\
52 & Penalty 1 (2nd version) & Moré, Garbow, and Hillstrom [12] & $1000,10,000$ \\
53 & INRIA u1ts0 & Gilbert and Lemaréchal [4] & 403 \\
\hline
\end{tabular}

the iteration count and the number of function-gradient evaluations, respectively. For the truncated-Newton method, this includes the number of gradient evaluations of the inner iteration (one gradient computation is considered to be as expensive as a simultaneous function and gradient evaluation). The times are measured in seconds and reflect total execution time, including function-gradient evaluations. The runs were made on one processor of an Encore Multimax computer, using Fortran in double precision (about 16 decimal digits). An upper bound of 9,999 function-gradient evaluations was imposed, but was only encountered by L-BFGS on test function one; this is indicated by a " + " next to the function-gradient count. Most of the test functions were run for two values of $n$. Table 4 presents the results for the smaller dimensions.

Table 5 gives the results for higher dimensions. On three runs, both routines terminated abnormally in the linesearch (a lower point could not be found). This is indicated by a "*." In all three cases the algorithms were close to the solution. These problems were rerun with the convergence tolerance in $(2.2)$ set to $10^{-5}$, to make it less stringent. With this change, these runs were successful and are included in Table 5 . As before, " + " indicates that the function evaluation limit was reached.

The results of Tables 4 and 5 are summarized in Table 6 . "Much better" is defined to be a difference of more than 30 percent in results. Differences of less than 10 percent were considered to be insignificant ("even"), as were absolute differences of less than one second and differences of less than five function-gradient evaluations. A failure is recorded as "much better" performance for the algorithm that succeeded.

What can we conclude from these results? First, neither algorithm is clearly superior to the other. Second, the limited-memory BFGS method tends to use fewer 
TABLE 4

Smaller test problems. Comparison of the limited memory BFGS method $(m=5)$ with the truncated-Newton method.

\begin{tabular}{lr|rrr|rrr}
\hline & & \multicolumn{3}{|c|}{ L-BFGS } & \multicolumn{3}{c}{ TN } \\
$\mathrm{P}$ & $\mathrm{N}$ & $\mathrm{It}$ & $\mathrm{f}-\mathrm{g}$ & Time & It & f-g & Time \\
\hline 1 & 100 & 9707 & +9999 & 2380.0 & 28 & 466 & 105.0 \\
2 & 100 & 1605 & 1669 & 304.0 & 27 & 242 & 41.4 \\
3 & 100 & 3095 & 3216 & 640.0 & 45 & 325 & 60.8 \\
6 & 100 & 257 & 291 & 19.0 & 78 & 683 & 35.3 \\
8 & 100 & 30 & 36 & 2.1 & 4 & 26 & 0.8 \\
9 & 100 & 21 & 23 & 2.4 & 8 & 48 & 4.4 \\
10 & 100 & 82 & 90 & 7.1 & 20 & 107 & 7.3 \\
28 & 100 & 57 & 67 & 3.6 & 14 & 70 & 3.0 \\
29 & 100 & 36 & 37 & 2.4 & 9 & 42 & 1.3 \\
31 & 100 & 24 & 27 & 10.7 & 14 & 101 & 38.5 \\
38 & 100 & 120 & 128 & 8.5 & 18 & 77 & 4.4 \\
39 & 121 & 66 & 70 & 8.4 & 18 & 187 & 19.6 \\
40 & 100 & 2219 & 2296 & 188.0 & 55 & 2091 & 146.0 \\
41 & 100 & 28 & 31 & 2.1 & 14 & 77 & 4.7 \\
42 & 1000 & 15 & 17 & 10.2 & 15 & 75 & 41.0 \\
43 & 100 & 19 & 21 & 1.7 & 10 & 38 & 2.9 \\
45 & 100 & 48 & 56 & 3.8 & 14 & 59 & 3.3 \\
$46(1)$ & 100 & 362 & 377 & 75.9 & 30 & 339 & 61.9 \\
$46(2)$ & 100 & 438 & 453 & 92.8 & 35 & 556 & 101.0 \\
47 & 100 & 87 & 95 & 10.1 & 17 & 94 & 8.8 \\
48 & 1000 & 38 & 49 & 26.4 & 16 & 79 & 32.0 \\
49 & 100 & 57 & 67 & 3.8 & 14 & 70 & 3.0 \\
50 & 100 & 129 & 133 & 9.5 & 17 & 78 & 4.2 \\
51 & 100 & 53 & 60 & 8.9 & 28 & 233 & 30.2 \\
52 & 1000 & 5 & 6 & 2.3 & 3 & 10 & 4.3 \\
\hline & & & & & & &
\end{tabular}

TABLE 5

Larger test problems. Comparison of the limited memory BFGS method $(m=5)$ with the truncated-Newton method.

\begin{tabular}{lr|rrr|rrr}
\hline & & \multicolumn{3}{|c|}{ L-BFGS } & & $\mathrm{TN}$ \\
$\mathrm{P}$ & $\mathrm{N}$ & $\mathrm{It}$ & $\mathrm{f}-\mathrm{g}$ & Time & It & $\mathrm{f}-\mathrm{g}$ & Time \\
\hline 1 & 200 & 9695 & +9999 & 5040.0 & 38 & 929 & 428.0 \\
2 & 200 & 1734 & 1785 & 646.0 & 37 & 456 & 154.0 \\
3 & 200 & 7248 & 7482 & 2960.0 & 76 & 599 & 220.0 \\
6 & 500 & 1054 & 1177 & 389.0 & 356 & 3446 & 796.0 \\
8 & 1000 & 30 & 34 & 21.0 & 12 & 58 & 23.9 \\
10 & 1000 & 103 & $* 114$ & 89.4 & 30 & $* 200$ & 142.0 \\
28 & 1000 & 54 & 61 & 35.8 & 15 & 75 & 34.6 \\
29 & 500 & 48 & $* 49$ & 15.4 & 12 & $* 54$ & 8.8 \\
31 & 200 & 3 & 4 & 5.8 & 4 & 20 & 24.9 \\
38 & 1000 & 405 & 423 & 302.0 & 33 & 208 & 121.0 \\
39 & 961 & 165 & 172 & 187.0 & 27 & 387 & 364.0 \\
42 & 10,000 & 14 & 17 & 102.0 & 24 & 111 & 696.0 \\
43 & 1000 & 16 & 20 & 15.4 & 15 & 75 & 59.1 \\
47 & 1000 & 145 & 157 & 168.0 & 22 & 160 & 145.0 \\
48 & 10,000 & 37 & 50 & 261.0 & 29 & 118 & 639.0 \\
49 & 1000 & 54 & 61 & 35.6 & 14 & 68 & 31.8 \\
50 & 1000 & 457 & 476 & 328.0 & 30 & 210 & 118.0 \\
51 & 1000 & 46 & $* 57$ & 80.1 & 35 & $* 370$ & 467.0 \\
52 & 10,000 & 6 & 8 & 32.5 & 5 & 19 & 90.9 \\
53 & 403 & 57 & 63 & 410.0 & 14 & 100 & 656.0 \\
\hline
\end{tabular}


TABLE 6

Summary of results: number of problems for which a method was better in terms of functiongradient evaluations and time.

\begin{tabular}{lrr}
\hline Result & f-g & Time \\
\hline L-BFGS much better & 18 & 21 \\
L-BFGS better & 6 & 1 \\
even & 8 & 4 \\
TN better & 2 & 1 \\
TN much better & 11 & 18 \\
\hline
\end{tabular}

function-gradient evaluations. Third, in terms of time, neither algorithm is a clear winner: the higher iteration cost of $\mathrm{TN}$ is compensated by a much lower iteration count, on the average. Fourth, the truncated-Newton method solved all the problems, whereas the limited memory BFGS method failed twice (on both versions of the first test problem). In $\S 5$ we analyze these results further, and present more detailed conclusions.

Tests of a simulated Newton method were also made. Algorithm TN was modified by changing the termination rule for the inner algorithm from (2.7) to

$$
\frac{\left\|G p_{i}+g_{k}\right\|_{2}}{\left\|g_{k}\right\|_{2}} \leq 10^{-8}
$$

and with an upper limit of $\min (n, 500)$ inner iterations imposed. Even though this does not represent a well-designed Newton method for large scale problems, it can indicate how many outer iterations would be required if the costs of the inner algorithm could be ignored. We refer to this simulated Newton method as SN. On the smaller test problems, SN required 313 iterations and TN required 551 ; in the worst case TN required five times more iterations. On the larger test problems, SN required 598 iterations and $\mathrm{TN}$ required 828; in the worst case $\mathrm{TN}$ required seven times more iterations. Hence, over the entire test set, TN required about 50 percent more iterations than SN. The L-BFGS method, whose iteration is much cheaper, typically requires between 3 and 10 times more iterations than $\mathrm{TN}$.

We conclude this section with a few comments on the partitioned quasi-Newton method of Griewank and Toint [8]. It assumes that the objective function is partially separable, i.e., that it can be written as

$$
f(x)=\sum_{i=1}^{n e} f_{i}(x),
$$

where the ne element functions $f_{i}$ depend only on a few variables. The partitioned quasi-Newton method (PQN) takes advantage of this structure of $f$ by updating a quasi-Newton approximation to each of the element functions $f_{i}$. Liu and Nocedal [10] compared L-BFGS and PQN, and concluded that when the number of variables entering into the element functions is very small (say, less than four), PQN is vastly superior to L-BFGS, both in terms of function evaluations and time. However, if the element functions $f_{i}$ depend on four or five variables, L-BFGS and PQN are often comparable. Furthermore, if more than five or six variables enter into the element functions, L-BFGS is likely to be more efficient.

4. Classification of the test problems. We would like to better understand the types of problems that each algorithm solves well. This immediately raises the 
question of how to classify the test problems. Among the various function characteristics that are relevant to the convergence theory or computational behavior of algorithms, we have selected the following.

(1) Deviation from quadratic. To assess this, we use the Taylor series approximation of the gradient, and define

$$
\mathrm{DQ}=\frac{\left\|g\left(x_{0}\right)-g\left(x^{*}\right)-G\left(x^{*}\right) p\right\|_{\infty}}{\|p\|_{\infty}^{2}},
$$

where $x_{0}$ and $x^{*}$ are the starting point and the solution, and $p=x_{0}-x^{*}$. Since we have scaled the difference by $\|p\|_{\infty}^{2}$, DQ gives a measure of the size of the third derivatives.

(2) Condition number of the Hessian. Using the $l_{2}$-norm, we measure the condition numbers of the Hessian at the starting point and solution point; these are denoted by $K_{0}$ and $K_{*}$, respectively.

(3) Convexity. This was sometimes determined by observation; otherwise we performed several computations to guide us. We computed the eigenvalues of the Hessian at $x_{0}$. If any were negative, the problem was clearly nonconvex. In addition, the inner algorithm of the truncated-Newton method can detect indefiniteness (although it is not guaranteed to find it). If none of these indicators suggested indefiniteness, and if we were not able to ascertain this theoretically, the problem was labeled "presumably convex."

(4) Eigenvalue structure. We plotted the eigenvalue distribution of the Hessian at the starting point and final point. The graphs are not given here due to space limitations, but can be obtained from the authors. The eigenvalue distribution greatly affects the performance of the inner conjugate-gradient algorithm of the truncated-Newton method, and Gill and Murray [6] suggest that it is also related to the efficiency of limited memory methods.

The characteristics of most of our test problems are displayed in Table 7 . We have only considered the case when $n \approx 100$, because the eigenvalue analysis for values of $n$ in the thousands is not tractable. In particular, test problem 42 was analyzed for $n=100$ rather than $n=1000$ (TN solved this smaller problem in 1.8 seconds using nine iterations and 35 function-gradient evaluations; L-BFGS used .9 seconds, 14 iterations, and 15 function-gradient evaluations). In Table 7, we also indicate which of the two methods performed best, and give the percentage difference in performance.

The next table has been arranged to show that there is a strong correlation between the degree of nonlinearity of the function and the success of the methods. We have concentrated on those problems of Table 7 in which the performance of the two methods is markedly different. Table 8 lists the problems for which one of the methods was better by at least 30 percent in terms of both time and function-gradient evaluations. The entries are ordered by degree of nonlinearity DQ.

We now list, in Table 9, three other properties of the test functions that help measure problem complexity, even though they do not appear to be directly useful for purposes of analysis.

(1) Sparsity. The degree of sparsity and the sparsity pattern determine whether Newton's method with sparse matrix techniques is attractive. Neither TN nor L-BFGS takes advantage of sparsity.

(2) Cost of evaluating $f$ and $g$. Here we merely timed the function values for most problems by averaging the time for 1000 function-gradient evaluations. For problem 53, only 20 evaluations were used in the timing because of the expense of computing the function. 
TABLE 7

Problem characteristics.

\begin{tabular}{lclllllrr}
\hline P & N & DQ & $1 / K_{0}$ & $1 / K_{*}$ & Convexity & Winner & Percent difference \\
& & & & & & & f-g & Time \\
\hline 1 & 100 & $10^{-2}$ & $10^{-8}$ & $10^{-8}$ & presumed & TN & 2045 & 2167 \\
2 & 100 & $10^{0}$ & $10^{-5}$ & $10^{-5}$ & presumed & TN & 590 & 634 \\
3 & 100 & $10^{0}$ & $10^{-6}$ & $10^{-6}$ & presumed & TN & 890 & 953 \\
6 & 100 & $10^{2}$ & $10^{-3}$ & $10^{-4}$ & no & L-BFGS & 135 & 86 \\
8 & 100 & $10^{2}$ & $10^{-1}$ & $10^{-2}$ & no & TN & 38 & 163 \\
9 & 100 & $10^{5}$ & $10^{-3}$ & $10^{-2}$ & presumed & L-BFGS & 109 & 83 \\
10 & 100 & $10^{2}$ & $10^{-2}$ & $10^{-3}$ & presumed & L-BFGS & 18 & 3 \\
28 & 100 & $10^{1}$ & $10^{-3}$ & $10^{-8}$ & presumed & even & 4 & 20 \\
29 & 100 & $10^{13}$ & $10^{-9}$ & $10^{-6}$ & no & even & 14 & 85 \\
31 & 100 & $10^{2}$ & 0 & $10^{-8}$ & presumed & L-BFGS & 274 & 260 \\
38 & 100 & $10^{-2}$ & 0 & 0 & yes & TN & 66 & 93 \\
39 & 121 & $10^{1}$ & $10^{-9}$ & 0 & no & L-BFGS & 167 & 133 \\
40 & 100 & $10^{-2}$ & $10^{-7}$ & $10^{-7}$ & no & TN & 10 & 30 \\
41 & 100 & $10^{1}$ & 0 & 0 & no & L-BFGS & 148 & 124 \\
42 & 100 & $10^{1}$ & $10^{-1}$ & $10^{-1}$ & presumed & L-BFGS & 133 & 100 \\
43 & 100 & $10^{0}$ & $10^{-3}$ & $10^{-4}$ & presumed & L-BFGS & 81 & 71 \\
45 & 100 & $10^{3}$ & $10^{-3}$ & $10^{-2}$ & no & even & 5 & 15 \\
$46(1)$ & 100 & $10^{1}$ & $10^{-4}$ & $10^{-4}$ & no & TN & 11 & 23 \\
$46(2)$ & 100 & $10^{1}$ & $10^{-3}$ & $10^{-4}$ & no & L-BFGS & 23 & 9 \\
47 & 100 & $10^{1}$ & $10^{-2}$ & $10^{-2}$ & no & even & 1 & 15 \\
49 & 100 & $10^{1}$ & $10^{-3}$ & $10^{-7}$ & presumed & even & 4 & 27 \\
50 & 100 & $10^{-4}$ & 0 & 0 & yes & TN & 58 & 126 \\
51 & 100 & $10^{2}$ & $10^{-3}$ & $10^{-2}$ & no & L-BFGS & 288 & 239 \\
53 & 403 & $10^{0}$ & 0 & 0 & no & L-BFGS & 59 & 60 \\
\hline
\end{tabular}

TABLE 8

Selected test problems, ordered by degree of nonlinearity.

\begin{tabular}{|c|c|c|c|c|c|}
\hline \multirow[t]{2}{*}{$\bar{P}$} & \multirow[t]{2}{*}{$\mathrm{N}$} & \multirow[t]{2}{*}{$\overline{\mathrm{DQ}}$} & \multirow[t]{2}{*}{ Winner } & \multicolumn{2}{|c|}{ Percent difference } \\
\hline & & & & $f-g$ & Time \\
\hline 9 & 100 & $10^{5}$ & L-BFGS & 109 & 83 \\
\hline 31 & 100 & $10^{2}$ & L-BFGS & 274 & 260 \\
\hline 8 & 100 & $10^{2}$ & $\mathrm{TN}$ & 38 & 163 \\
\hline 6 & 100 & $10^{2}$ & L-BFGS & 135 & 86 \\
\hline 51 & 100 & $10^{2}$ & L-BFGS & 288 & 239 \\
\hline 41 & 100 & $10^{1}$ & L-BFGS & 148 & 124 \\
\hline 42 & 100 & $10^{1}$ & L-BFGS & 133 & 100 \\
\hline 39 & 121 & $10^{1}$ & L-BFGS & 167 & 133 \\
\hline 3 & 100 & $10^{0}$ & $\mathrm{TN}$ & 890 & 953 \\
\hline 2 & 100 & $10^{0}$ & $\mathrm{TN}$ & 590 & 634 \\
\hline 43 & 100 & $10^{0}$ & L-BFGS & 81 & 71 \\
\hline 53 & 403 & $10^{0}$ & L-BFGS & 59 & 60 \\
\hline 1 & 100 & $10^{-2}$ & $\mathrm{TN}$ & 2045 & 2167 \\
\hline 38 & 100 & $10^{-2}$ & $\mathrm{TN}$ & 66 & 93 \\
\hline 50 & 100 & $10^{-4}$ & $\mathrm{TN}$ & 58 & 126 \\
\hline
\end{tabular}

(3) Laboriousness. This is an attempt to measure the difficulty of solving a problem. We considered both algorithms, and recorded the minimum number of function-gradient evaluations and the minimum time to solve the problem.

One final point about the test problems should be made. Of those analyzed here, 13 out of 24 (and all eight of the most laborious problems) are singular, nearly singular, or nonconvex. The fact that all these problems were successfully solved by both methods seems to indicate that their implementations are very robust. 
TABLE 9

Other problem characteristics.

\begin{tabular}{lrlrrrrrr}
\hline P & N & Sparsity & Cost & \multicolumn{2}{c}{ Laboriousness } & Winner & \multicolumn{2}{r}{ Percent difference } \\
& & & & f-g & Time & & Time \\
\hline 1 & 100 & 7-diagonal & .155 & 466 & 105.0 & TN & 2045 & 2167 \\
2 & 100 & 7-diagonal & .123 & 242 & 41.4 & TN & 590 & 634 \\
3 & 100 & 7-diagonal & .121 & 325 & 60.8 & TN & 890 & 953 \\
6 & 100 & tridiagonal & .014 & 291 & 19.0 & L-BFGS & 135 & 86 \\
8 & 100 & dense & .011 & 26 & 0.8 & TN & 38 & 163 \\
9 & 100 & dense & .054 & 23 & 2.4 & L-BFGS & 109 & 83 \\
10 & 100 & dense & .026 & 90 & 7.1 & L-BFGS & 18 & 3 \\
28 & 100 & $4 \times 4$ block diagonal & .007 & 67 & 3.0 & even & 4 & 20 \\
29 & 100 & dense & .009 & 37 & 1.3 & even & 14 & 85 \\
31 & 100 & dense & .322 & 27 & 10.7 & L-BFGS & 274 & 260 \\
38 & 100 & tridiagonal & .014 & 77 & 4.4 & TN & 66 & 93 \\
39 & 121 & 9 non-zero diagonals & .054 & 70 & 8.4 & L-BFGS & 167 & 133 \\
40 & 100 & 5-diagonal & .026 & 2091 & 146.0 & TN & 10 & 30 \\
41 & 100 & 5-diagonal & .018 & 31 & 2.1 & L-BFGS & 148 & 124 \\
42 & 100 & tridiagonal & .013 & 15 & 0.9 & L-BFGS & 133 & 100 \\
43 & 100 & tridiagonal & .032 & 21 & 1.7 & L-BFGS & 81 & 71 \\
45 & 100 & tridiagonal & .015 & 56 & 3.3 & even & 5 & 15 \\
$46(1)$ & 100 & dense & .137 & 339 & 61.9 & TN & 11 & 23 \\
$46(2)$ & 100 & dense & .131 & 453 & 92.8 & L-BFGS & 23 & 9 \\
47 & 100 & 9-diagonal & .046 & 94 & 8.8 & even & 1 & 15 \\
49 & 100 & 4 $\times 4$ block diagonal & .009 & 67 & 3.0 & even & 4 & 27 \\
50 & 100 & tridiagonal & .011 & 78 & 4.2 & TN & 58 & 126 \\
51 & 100 & dense & .094 & 60 & 8.9 & L-BFGS & 288 & 239 \\
53 & 403 & dense & 5.980 & 63 & 410.0 & L-BFGS & 59 & 60 \\
\hline & & & & & & & & \\
\end{tabular}

5. Further analysis of the numerical results. Using the tables of results and of function characteristics of the previous sections, we now try to correlate the types of test problems with the success of TN and L-BFGS. Since we have seen that in terms of time the two methods are similar, we concentrate on the number of function-gradient evaluations.

The first, very visible, trend is that the performance of the two algorithms appears to be correlated with the degree of nonlinearity DN: for quadratic and approximately quadratic problems, TN outperforms L-BFGS. In fact, TN was better almost exclusively for these types of problems. Among the 11 problems for which TN was considered "much better" in Table 6, four are quadratic (both versions of problems 38 and 50) and six are approximately quadratic (both versions of problems 1,2 , and $3)$. Moreover, one of the two problems for which TN was considered "better" in this table is approximately quadratic (problem 40). For most of the highly nonlinear problems, L-BFGS performed better. It appears that TN's effort to approximate the Newton step is not paying off on highly nonlinear problems. Continuing work on trying to improve the performance of $\mathrm{TN}$ by reducing the number of inner iterations on problems that are detected to be highly nonlinear will be reported in a future paper [17].

A study of the eigenvalue distribution of the Hessian matrices shows no clear correlation between the success of the methods and the eigenvalue structure. For problems with ill-conditioned Hessians, $\mathrm{TN}$ is perhaps better, but this is difficult to ascertain from our tests. Clustering of eigenvalues at the solution does not seem to benefit one method more than the other. There is little or no correlation between the other measures of the test problems and the performance of the two methods.

L-BFGS did very poorly on the calculus of variations problems 1,2 , and 3 . These 
problems are not only ill conditioned; their Hessians have many eigenvalues near zero (for problem 1 for $n=100$, about half the eigenvalues are near zero). We ran LBFGS with a more accurate linesearch and using more memory, and even though the performance improved substantially, it was still far from competitive with TN. For example, using $m=20$ corrections and setting $\eta=10^{-3}$ in (2.3) resulted in 418 iterations, 861 function-gradient evaluations, and 183 seconds for problem 2 with $n=100$. The calculus of variations problems also caused difficulties for earlier versions of the TN method that used no preconditioning; the performance was as bad as for L-BFGS. If $n$ is increased to 1000 , TN has trouble solving the problem; however, block versions of the TN method (requiring greater storage and work per iteration) are capable of solving it [15]. We should note that Newton's method is very effective on this problem, suggesting that an accurate approximation to the Newton direction is needed.

It appears from this test set that, in terms of function evaluations, L-BFGS is preferable to $\mathrm{TN}$ for more highly nonlinear (not approximately quadratic) problems. However, TN almost always requires many fewer iterations than L-BFGS, and therefore, if the number of gradient evaluations in the inner iteration could be significantly reduced, TN would be competitive or more efficient than L-BFGS. How to realize these savings is a question that deserves further investigation. It does not appear that automatic differentiation (see, for example, [7]) will help, because the cost is of the same order as that of the gradient differences used here. Differencing along selective directions determined by the sparsity of the problem could be useful, but in this case both Newton's method with sparse matrix techniques and the partitioned quasi-Newton method may be preferable.

6. The nonlinear conjugate-gradient method. Now that we have studied the relative performances of L-BFGS and TN, we will use another, well-established, algorithm to measure their efficiency. To this end, we will solve our set of test problems with the Polak-Ribière version of the conjugate-gradient method, which is one of the classical methods for solving large problems. We chose the Polak-Ribière method for the following reason.

More recent implementations of the conjugate-gradient method, like the Harwell routine VA14 of Powell [21] or the routine CONMIN of Shanno and Phua [22], which include automatic restarts, and store additional information, require fewer function evaluations than the Polak-Ribière method. However, Liu and Nocedal [10] found that L-BFGS clearly outperforms CONMIN, both in terms of computer time and function evaluations - and it is known that CONMIN is more efficient than VA14. On the other hand, the Polak-Ribière method appeared to be often competitive with LBFGS in terms of computer time (but not in terms of function evaluations). Therefore, we chose the Polak-Ribière method for this study, since it appears to be the only implementation of the conjugate-gradient method that could be competitive (at least in terms of computer time) with TN and L-BFGS.

The Polak-Ribière iteration is

$$
x_{k+1}=x_{k}+\alpha_{k} p_{k}
$$

where the steplength $\alpha_{k}$ satisfies the strong Wolfe conditions (2.3)-(2.4), and where

$$
p_{k}=-g_{k}+\beta_{k} p_{k-1},
$$


TABLE 10

Smaller problems. Results of the Polak-Ribière conjugate-gradient method (CG).

\begin{tabular}{lrrrr|cc|cc}
\hline & \multicolumn{3}{c|}{ CG } & \multicolumn{2}{c|}{ L-BFG } & \multicolumn{2}{c}{ TN } \\
\hline $\mathrm{P}$ & $\mathrm{N}$ & $\mathrm{It}$ & $\mathrm{f}-\mathrm{g}$ & Time & f-g & Time & f-g & Time \\
\hline 1 & 100 & 4915 & +9999 & 1910.0 & $\approx$ & $\approx$ & & \\
2 & 100 & 656 & 1506 & 201.0 & + & $*$ & & \\
3 & 100 & 2082 & 4243 & 641.0 & & $\approx$ & & $*$ \\
6 & 100 & 275 & 1129 & 24.2 & & & & $\approx$ \\
8 & 100 & 9 & 28 & 0.6 & + & $*$ & & \\
9 & 100 & $\mathrm{~F}$ & & & & & & \\
10 & 100 & 119 & 304 & 11.3 & & & & \\
28 & 100 & 108 & 277 & 4.7 & & $*$ & & $\approx$ \\
29 & 100 & 7 & 51 & 1.0 & & $*$ & + & $*$ \\
31 & 100 & 3 & 12 & 4.3 & + & $*$ & & $\approx$ \\
38 & 100 & 76 & 154 & 3.8 & & & $\approx$ & $*$ \\
39 & 121 & 63 & 180 & 12.6 & & & & \\
40 & 100 & 5001 & +9999 & 363.0 & & & \\
41 & 100 & 27 & 63 & 1.8 & & $\approx$ & + & $*$ \\
42 & 1000 & 14 & 37 & 8.0 & & & + & $*$ \\
43 & 100 & 54 & 141 & 7.1 & & $*$ & & $\approx$ \\
45 & 100 & 36 & 120 & 2.8 & & & & \\
$46(1)$ & 100 & 286 & 583 & 89.3 & & & & $\approx$ \\
$46(2)$ & 100 & 313 & 639 & 99.4 & & $\approx$ & & $\approx$ \\
47 & 100 & 73 & 152 & 9.6 & & $\approx$ & & $*$ \\
48 & 1000 & 25 & 108 & 14.2 & & & & \\
49 & 100 & 104 & 255 & 4.8 & & $*$ & & $\approx$ \\
50 & 100 & 79 & 160 & 3.6 & & & + & $*$ \\
51 & 100 & 54 & 114 & 12.1 & & & $*$ \\
52 & 1000 & 4 & 10 & 1.6 & $\approx$ & $\approx$ & $\approx$ & $*$ \\
\hline
\end{tabular}

with

$$
\beta_{k}=\frac{y_{k-1}^{T} g_{k}}{\left\|g_{k-1}\right\|^{2}} .
$$

The linesearch is performed by means of the routine of Moré and Thuente [11], previously mentioned in $\S 2$, with two small changes: (i) Since the Wolfe conditions do not ensure that descent directions are always generated, we continue the linesearch iteration until the descent condition is guaranteed. (ii) We insist that the linesearch performs at least one quadratic (or cubic) interpolation, and hence the algorithm reduces to the linear conjugate-gradient algorithm if the objective function is quadratic.

We chose $\mu=10^{-4}$ and $\eta=0.1$, for the parameters in (2.3)-(2.4), because our earlier experience with the Polak-Ribière method indicated that these values give the best results. The stopping condition was (2.2). The tests were performed, as before, on an Encore Multimax. The algorithm was restarted every $n$ iterations by setting $\beta_{k}=0$, which ensures global convergence in exact arithmetic. We note that $n$ is often larger than the number of iterations, so that the algorithm performs no restarts in many of our test runs. The Polak-Ribière method requires $4 n$ storage locations, and as shown by (6.2)-(6.3), the computation of $d_{k}$ is very inexpensive.

Tables 10 and 11 present the results of the Polak-Ribière (CG) method on the whole collection of test problems. Under the column labelled "CG" we give the number of iterations/number of function-gradient evaluations, and the total computing time. As before, a "+" indicates that the function evaluation limit was reached, and a "*" indicates that the weaker convergence test (described in the third paragraph of $\S 3)$ was used. In the runs marked " $F$ " the search direction was so out of scale that 
TABLE 11

Larger problems. Results of the Polak-Ribière conjugate-gradient method (CG).

\begin{tabular}{lrrrr|lc|cc}
\hline & \multicolumn{5}{c|}{ CG } & \multicolumn{2}{c|}{ L-BFGS } & \multicolumn{2}{|c}{ TN } \\
\hline $\mathrm{P}$ & $\mathrm{N}$ & $\mathrm{It}$ & $\mathrm{f}-\mathrm{g}$ & Time & f-g & Time & f-g & Time \\
\hline 1 & 200 & 4804 & +9999 & 3840.0 & $\approx$ & $\approx$ & & \\
2 & 200 & 1106 & 2491 & 657.0 & & $\approx$ & & \\
3 & 200 & 3336 & 6847 & 2060.0 & $\approx$ & $*$ & & $*$ \\
6 & 500 & 1098 & 4889 & 515.0 & & & & $*$ \\
8 & 1000 & 13 & 36 & 7.3 & $\approx$ & $*$ & + & $*$ \\
10 & 1000 & & $* \mathrm{~F}$ & & & & & \\
28 & 1000 & 167 & 456 & 72.1 & & & & $*$ \\
29 & 500 & 8 & $* 98$ & 7.1 & & $*$ & & $*$ \\
31 & 200 & 3 & 14 & 20.3 & & & + & $*$ \\
38 & 1000 & 285 & 573 & 138.0 & & $*$ & & $*$ \\
39 & 961 & 161 & 519 & 319.0 & & & & $*$ \\
42 & 10000 & 11 & 33 & 68.9 & & $*$ & + & $*$ \\
43 & 1000 & 16 & 56 & 23.3 & & & + & $*$ \\
47 & 1000 & 145 & 302 & 181.0 & & $\approx$ & & $*$ \\
48 & 10000 & 18 & 76 & 103.0 & & $*$ & + & $*$ \\
49 & 1000 & 225 & 615 & 104.0 & & & & \\
50 & 1000 & 294 & 591 & 116.0 & & $*$ & & $\approx$ \\
51 & 1000 & 41 & $* 91$ & 93.7 & & & + & $*$ \\
52 & 10000 & 5 & 13 & 21.0 & $\approx$ & $*$ & + & $*$ \\
53 & 403 & 62 & 144 & 926.0 & & & & \\
\hline
\end{tabular}

overflow occurred. Additional safeguarding could remedy these failures, but was not implemented. We compare the performance of CG with that of L-BFGS and TN by indicating the problems for which CG was more effective. In the column labelled "L-BFGS," a "+" in the column "f-g" indicates that CG required fewer function calls, and $a$ "*" in the column "Time" indicates that it required less computing time than L-BFGS. The sign " $\approx$ " means equal or near-equal performance (measured in the same way as in Table 6: differences of less than 10 percent, less than one second, or less than five gradient evaluations were considered negligible). The same notation is used in the column labelled "TN." We verified, for each run, that the solution point obtained by CG coincided with that found by L-BFGS and TN.

It is clear that the conjugate-gradient method is not competitive with $\mathrm{TN}$ or L-BFGS in terms of function evaluations. However, it is often efficient in terms of computing time, due to its very low iteration cost. From this set of test runs, we conclude that the conjugate-gradient method is not to be recommended when the function evaluation is expensive, but that it may be useful for very large problems whose objective functions are relatively inexpensive.

Acknowledgments. We would like to thank the referees for several valuable comments and suggestions.

\section{REFERENCES}

[1] A. BuCKLey AND A. LeNir (1983), QN-like variable storage conjugate gradients, Math. Programming, 27, pp. 155-175.

[2] T.F. ColEman AND J. MoRÉ (1984), Estimation of sparse Hessian matrices and graph coloring problems, Math. Programming, 28, pp. 243-270.

[3] P. Concus, G. Golub, AND D. P. O'LeARy (1976), A generalized conjugate gradient method for the numerical solution of elliptic partial differential equations, in J. Bunch and D. Rose, eds., in Sparse Matrix Computations, Academic Press, New York, pp. 309-332. 
[4] J. C. Gilbert AND C. LEMARÉChAL (1989), Some numerical experiments with variable storage quasi-Newton algorithms, Math. Programming, 45, pp. 407-435.

[5] P. E. Gill AND W. MURRAY (1973), The numerical solution of a problem in the calculus of variations, in Recent Mathematical Developments in Control, D.J. Bell, ed., Academic Press, New York, pp. 97-122.

[6] - (1979), Conjugate-gradient methods for large-scale nonlinear optimization, Report SOL 79-15, Department of Operations Research, Stanford University, Stanford, CA.

[7] A. Griewank (1989), On automatic differentiation, in Mathematical Programming, M. Iri and K. Tanabe, eds., Kluwer Academic Publishers, Tokyo, pp. 83-107.

[8] A. GRIEwank AND PH. L. ToInt (1982), Partitioned variable metric updates for large structured optimization problems, Numer. Math., 39, pp. 119-137.

[9] D. C. LIU AND J. NocedAL (1988), Test results of two limited memory methods for large scale optimization, Report NAM 04, Department of Electrical Engineering and Computer Science, Northwestern University, Evanston, Illinois.

[10] - (1989), On the limited memory BFGS method for large scale optimization, Math. Programming, 45, pp. 503-528.

[11] J. J. MORÉ, AND D. J. ThUENTE (1990), On linesearch algorithms with guaranteed sufficient decrease, Mathematics and Computer Science Division Preprint MCS-P153-0590, Argonne National Laboratory, Argonne, IL.

[12] J. J. MorÉ, B. S. Garbow, AND K. E. Hillstrom (1981), Testing unconstrained optimization software, ACM Trans. Math. Software, 7, pp. 17-41.

[13] S. G. NASH (1984), User's guide for TN/TNBC: Fortran routines for nonlinear optimization, Report 397, Mathematical Sciences Department, The Johns Hopkins University, Baltimore, MD.

[14] -(1985), Preconditioning of truncated-Newton methods, SIAM J. Sci. Statist. Comput., 6, pp. 599-616.

[15] S. G. NASH AND A. Sofer (1989), Block truncated-Newton methods for large-scale nonlinear optimization, Math. Programming, 45, pp. 529-546.

[16] (1990a), Assessing a search direction within a truncated-Newton method, Oper. Research Lett., 9, pp. 219-221.

[17] - (1990b), A practical truncated-Newton method for parallel optimization, Report 63, Center for Computational Statistics, George Mason University, Fairfax, VA.

[18] J. Nocedal (1980), Updating quasi-Newton matrices with limited storage, Math. Comp., 35, pp. $773-782$.

[19] D. P. O'LEARY (1983), A discrete Newton algorithm for minimizing a function of many variables, Math. Programming, 23, pp. 20-33.

[20] S. OREN AND E. SPEDICATO (1976), Optimal conditioning of self-scaling variable metric algorithms, Math. Programming, 10, pp. 70-90.

[21] M. J. D. Powell (1977), Restart procedures for the conjugate gradient method, Math. Programming, 12, pp. 241-254.

[22] D. F. Shanno AND K. H. PhuA (1980), Remark on algorithm 500: Minimization of unconstrained multivariate functions, ACM Trans. Math. Software, 6, pp. 618-622.

[23] Ph.L. ToINT (1983), Test problems for partially separable optimization and results for the routine PSPMIN, Report Nr 83/4, Department of Mathematics, Facultés Universitaires de Namur, Namur, Belgium. 